Anat. Labor, of Prof. H. SETO, Tohoku University, Sendai.

\title{
On the Histology and the Sensory Innervation of the Vagina and the Sinus Urogenitalis of Dog.
}

犬銐及び㲾性器洞の組織と神経分布とと就て。

Yukihiko MIURA 三浦幸彦。

(Received February 6, 1956.)

Recently, KATO of this laboratory has succeeded in shedding light on the histology of the clitoris of dog and in obtaining many very interesting findings on the innervation, especially the sensory innervation of this part. I. felt much interested in his study, and beside the sections prepared by KATO under SETO's silver impregnation method, made my own transverse sections of the same part under a similar manner, which I subjected to minute examination. By this, I could not only confirm the findings of KATO on the dog clitoris without reserve, but also bring the histological pictures of the sinus urogenitalis and of the vagina and the urethra coming together in it to light and elucidate their innervation, especially, the sensory innervation, as reported in the following.

\section{Individual Findings.}

In his report on the clitoris of dog, KATO has schematically illustrated the median section of the feminine outer genitals of $\operatorname{dog}$ in his Fig. 8, well illuminating the topography of these organs. The part of it depicting the sinus urogenitalis and the vagina, however, is subject to some revision, according to what $I$ have found in my study.

Now, I have found the following marked morphological difference according to the position in the lumina of the sinus urogenitalis and the vagina. At the distal end of the sinus, its lumen, as is revealed upon examination of transverse sections of the part, shows the form of a narrow split extending dorsoventralwards, only $2-3$ small short furrows being formed at the mid-part of the two sides. Thus, the lumen is here in the form of a tall but narrow one (Fig. 1). Going more cranialwards to its intermediate part, the lumen of the sinus decreases in height but becomes broader and approximately rhomboid in cross-section (Fig. 2), with the vertices directed dorsoventrally and bilaterally. Upon reaching more cranially the vicinity of the opening of the urethra, the lumen becomes compressed dorsoventrally into a form of meniscus with its concave side facing ventralwards, so that the height becomes very low. Here, 3 or 4 short furrows are found on the dorsal wall, but the 


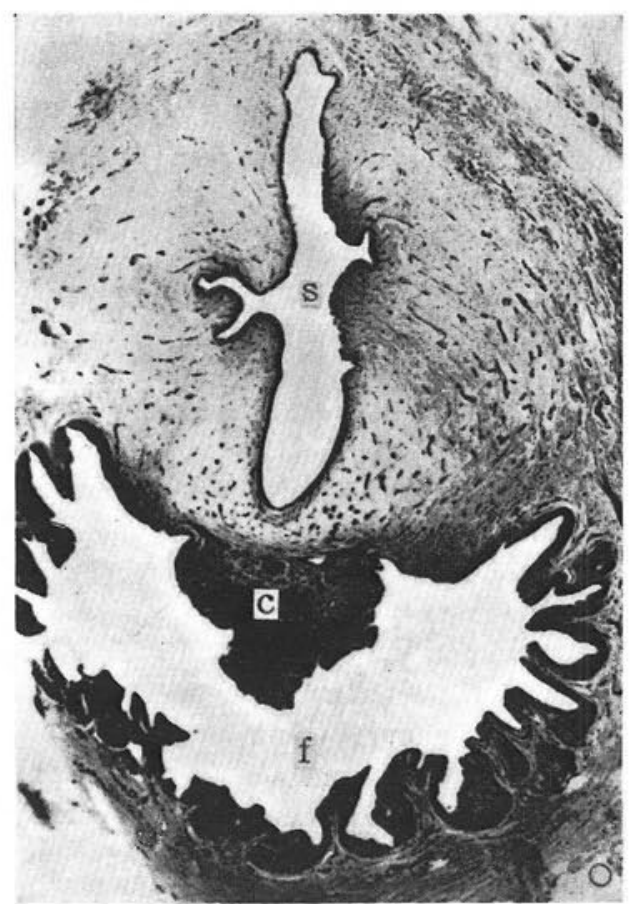

Fig. 1. A frontal section through the distal part of the sinus urogenitalis of a femine dog. $s$ long and slender lumen of the sinus, $c$ clitoris, $f$ fossa clitoridis. SETO's impregnation.

Photo $\times 6$.

mucous membrane lining the other sides is smooth-surfaced throughout (Fig. 3).

Passing over from the sinus into the vagina, the meniscus becomes more curved and the lumen even more narrow, laking the form of a split. The surface of the mucous membrane is even and nearly quite smooth, only one single shallow furrow dug into the dorsal wall of the lumen (Fig. 4). When the lumen of the vagina is followed up more cranially, the curvature of the meniscus grows gradually lighter while low furrows formed by depression of the mucous membrane begin to be perceptible here and there. The meniscoid cross-section of the lumen gradually lowering in curvature finally changes into a form of a horizontal slit and the furrows increase to about 15 in number and become better developed, some showing formation of secondary furrows (Fig. 5). This part, of the vagina with furrows and folds is the essenstial part of it, the part lined by a smooth-surfaced mucous membrane seen more 


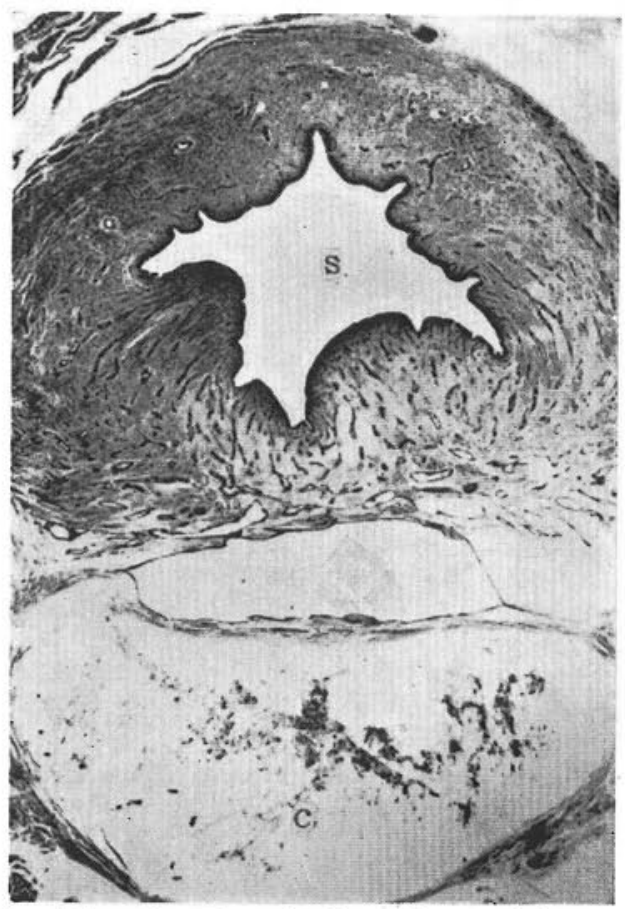

Fig. 2. A frontal section through the mid-part of the sinus urogenitalis of a femine dog. $s$ rhomboid lumen of the sinus, $c$ corpus clitoridis composed of fat tissue. Same staining. Photo $\times 6$.

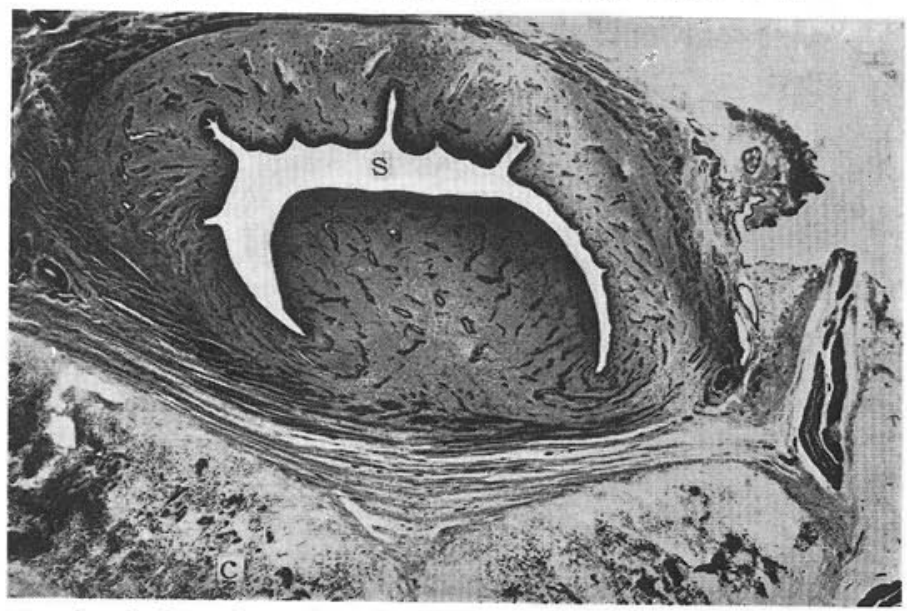

Fig. 3. A frontal section through the proximal part of the sinus urogenitalis of a femine dog. $s$ semilunar lumen of the sinus with a few furrows, $c$ corpus clitoridis. Same staining. Photo $\times 6$. 


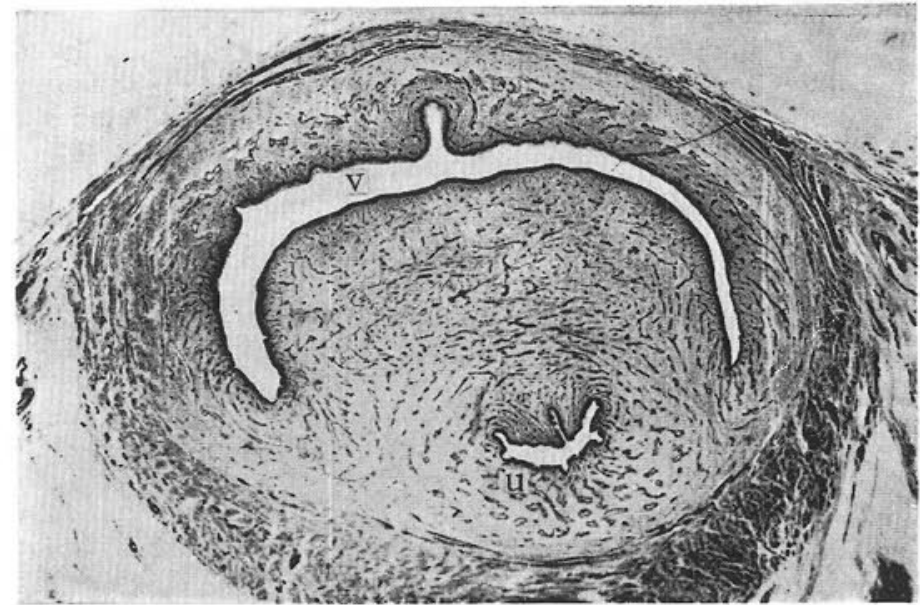

Fig. 4. A frontal section through the distal part of the dog vagina. $v$ semilunar lumen with a short dorso-median furrow, $u$ urethra devoid with the muscularis. Same staining. Photo $\times 6$.

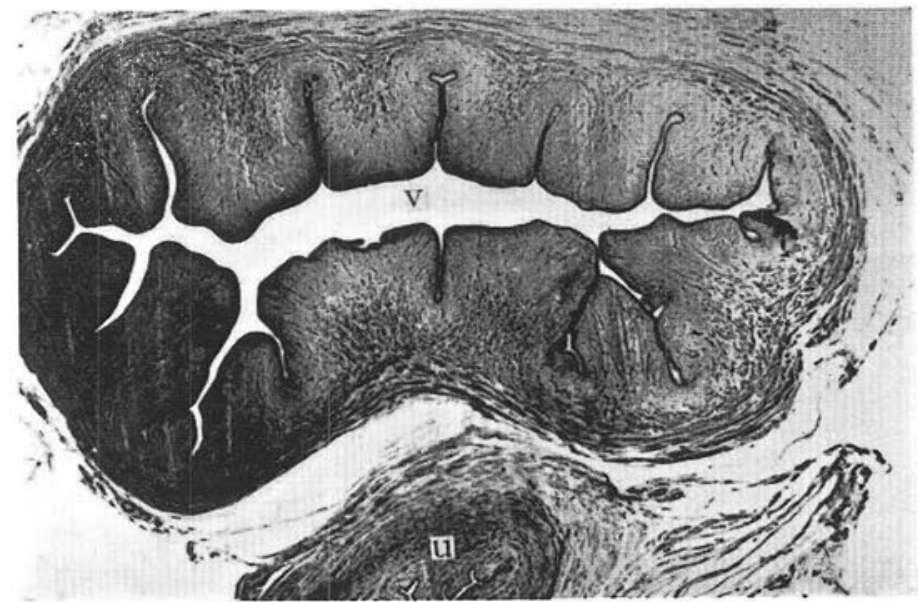

Fig. 5. A frontal section through the proper vagina of a dog. $v$ horizontal lumen with many long furrows, $m$ muscularis divided into the inner longitudinal and the outer circular smooth muscle layers, $u$ urethra provided with a powerful muscularis. Same staining. Photo $\times 6$.

caudally being a part rather similar to the sinus urogenitalis in nature. This may be readily understood when you come to the histological description of this part below. Fig. 6 shows the schema of the median section of the vagina and the sinus urogenitalis I have drawn up em- 


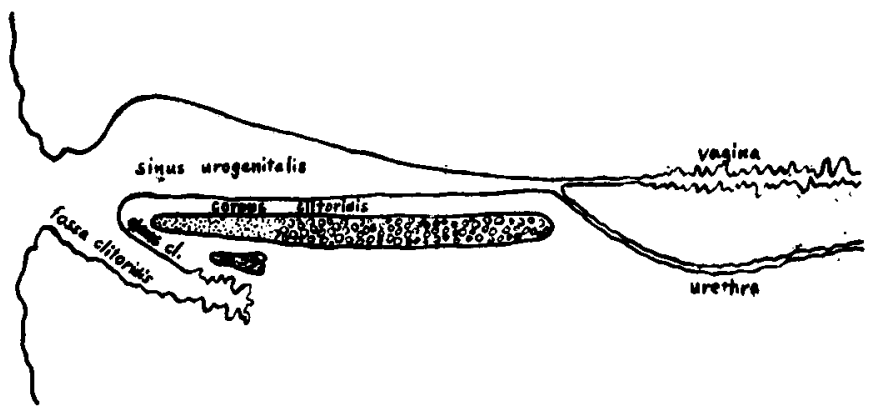

Fig. 6. A schema of the femine outer genitals in dog through the median section of the sinus urogenitalis and the vagina.

bodying the results of my study.

As detailed above, the lumen of the sinus urogenitalis and the vagina shows a morphological variation according to the position - a very interesting finding. It must be also well noted that, although the lumen shows a very peculiar change in the form of the cross-section, between the distal end of the sinus and that of the vagina, the mucous membrane lining that part of the lumen shows little change in its whole circumference, but the mucous membrane with many furrows and consequently many mucous folds in the main part of vagina has the circumference around the lumen of 3 to 4 times of that in the above.

It is of great interest that the cross-section of the lumen of the vagina and of the sinus urogenitalis changes in unison with the difference of their histological structure, as detailed below, and also that the change in form is closely related with the distribution of the sensory fibres. I will first give a description on the histological structure and then on the sensory innervation.

In the vagina proper $r i c h$ in furrows and folds, the epithelium consists of non-cornified stratified flat epithelium consisting of several layers of epithelial cells, but in the deeper parts of the furrows, it sometimes takes the form of cylindric epithelium. The propria consists of a thin layer of fibrous connective tissue rich in fibrocytes and contains rather abundant blood capillaries. Infiltration of lymphocytes, occasionally found in human vagina, is never observable in dog. The development of the papillae formed against the epithelium from the propria is also very poor.

The submucosa consists of a very thick layer formed by an assemblage of fibrocytes much poorer in quantity than in the propria and of thick collagenous fibres. These fibres, in a transverse section, run in a manner as if encircling the furrows but in a perpendicular direction against the folds. Rather large blood vessels are seen running in and 
out of the submucosa, but unlike in man, no venous plexus is formed therein. Around this well-developed submucosa is seen a muscularis formed in loose arrangement, which consists generally of an inner longitudinal and an outer circular layers, except in the dorsal wall of the vagina, where a third ill-developed longitudinal layer is formed. The inner longitudinal layer is formed more strongly in the basal parts of the folds than in those of the furrows. The circular layer is worse developed on the dorsal side where a part of it is substituted by longitudinal muscle fibres. The muscularis is lined on both sides by a thin adventitia of connective lissue and butts on the urethra on the ventral side. The striated muscle layer lining the urethra stretches to the lateral sides of the vagina and thence further to the dorsal, but here its development is much poorer.

When the caudal part of the vagina where the development of the folds loses in power, the histological picture begins to show an interesting change. The epithelium is here thicker on the dorsal than on the yentral wall and venous plexus begin to appear in gradually increasing number in the submucosa, while the muscular layer slowly loses in development, especially on the dorsal side.

The urethra running along the ventral side of the vagina proper rich in mucous folds consists of a 2-5 rowed cylindrical epithelium, a thin propria, a thick submucosa, an inner longitudinal and an outer circular smooth muscle layers and then of a well-developed circular striated muscle layer lining the outside of these layers, so it is almost identical in structure with the vagina. In the submucosa of this urethra, however, venous plexus formation is already observable, unlike in the vagina. The vagina and the urethra are loosely connected by a common adventitia.

As the vagina comes to have a semilunar lumen, the urethra takes the course of as if shut in this half cylinder and approximates the median line of the concave side of it as it proceeds distalwards. Thus the wall of the urethra seems to become absorbed in the tissue of the ventral wall of the vagina and the adventitia separating the two first vanishes, then the muscularis of the urethra disappears and the submucosae of the urethra and the vagina of similar nature fuse together naturally enough. Here, venous plexuses come into formation in the vaginal wall too, and in the distal part it is even rather full of them. The smooth muscle layer of the vagina loses also gradually in development, so that in the outermost part of the vagina, smooth muscle fibres remain only in a few longitudinal or circular strands. The striated muscle layer lining the outermost side of the wall also diminishes considerably in thickness, especially on the dorsal side. The epithelium is usually rather 
thick, except in the parts lining the vertices of the semilunar lumen, where it changes into a thin double-rowed cylindrical epithelium.

The histological picture of the proximal one-third of the sinus urogenitalis provided with semilunar lumen is approximately identical with that of the distal part of the vagina detailed in the above. The epithelium of this part, however, is considerably thinner on the ventral wall of the meniscus and the small longitudinal smooth muscle bundles arranged loosely along the outside of the median part of the submucosa of the dorsal side gain gradually in quantity. The sinus urogenitalis touches the corpus clitoridis surrounded by a connective tissue on the ventral side.

In the middle one-third of the sinus urogenitalis with rhomboidform lumen, the epithelium is either of flat or cylindrical type, which is generally thin on the ventral side, especially at the ridges of the furrows. The thin propria is rich in capillaries here too, and lymphocyte gatherings appear not rarely in this part. Venous plexus is still found in formation, though only in the ventral half. This plexus is connected with the plexus consiting of very stout veins and found in the adventitia. The latter passes over into the similarly well-developed venous plexus in fat tissue lying further outside. In the dorsal half of the submucosa, however, the venous plexus shows the appearance of total absence, its place in the outer layer of the submcosa being taken by many loosely arranged narrow bundles of longitudinal smooth muscle fibres.

The histological picture of the most distal part of the sinus urogenitalis facing the fossa clitoridis where the glans clitoridis is visible and the lumen is elongated ventrodorsally also changes gradually as the distal end is approached. The epithelium grows thicker dorsalwards and thinner ventralwards and lymphatic follicles are found scattered in the subepithelial tissue, as is the case with the mid-part of the sinus, but the venous plexus found there in the submucosa gradually vanishes distalwards while the small longitudinal smooth muscle bundles become worse developed and finally disappear.

As is the above, the feminine outer genital of dog shows a wide. morphological difference from that of man but it may be understood that the difference is not of an essential nature, because, though in dog, the vagina and the urethra have their outer openings joined in the long mucous canal of the sinus urogenitalis and the, glans clitoridis is not superficially visible but is formed in the mucous membrane of a deep fossa clitoridis, this sinus urogenitalis corresponds in essence to the human vestibulum vaginae. This physiological identity of the sinus and the vestibulum vaginae may be readily grasped from the results of my 
study on the innervation, especially, the sensory innervation of the former as detailed in the following.

According to the report of IKUI, the human vagina is scarcely supplied with sensory nerves, a few branched sensory terminations being observed only near its outer orifice. Sensory terminations are said to be more abundant in the vestibulum vaginae adjoining the orifice and the sensory innervation is further forlified in the labia minora where many corpuscular terminations such as genital nerve bedies are also found.

In my sections of the feminine outer genitals of dog, the sensory jnnervation was found to show similar findings as that in human counterparts reported by IKUI, if only the canine sinus urogenitalis is identified with the human vestibulum vaginae - an observation of deep interest indeed.

The nerves in the vagina proper of dog provided with well-developed furrows and folds come from the plexus vaginalis found in the adventitia containing small ganglia and originating in the plexus uterovaginalis. Thus, the part is very well supplied with vegetative fibres, but in this plexus scarcely any sensory fibre could be demonstrated. Therefore, the mucous membrane of the dog vagina proper is found free of sensory nerve supply. In the distal part of the vagina with a semilunar lumen and lined by a foldless mucous membrane, however, as the surrounding smooth muscle layer rapidly falls off in development, the formation of the plexus vaginalis also becomes weaker, but the $n$. perinealis originating in the $\mathrm{n}$. pudendalis makes appearance on the ventrolateral side and fine branches sent out by the former run through the adventitia inwards, to diffuse out in the mucous membrane. Thus, the distal part of the vagina of $d o g$ is as well supplied with sensory fibres as the human counterpart, and it is of interest that the sensory elements are even rather widely better developed in dog than in man. On the ventral side of the distal end of the vagina the corpus elitoridis formed by a fat tissue extending dorsalwards begins to appear, and at the same time, the stout $n$. dorsalis clitoridis begins to run distally along the ventral side of the corpus.

The sinus urogenitalis formed by the union of the vagina and the urethra is also supplied with sensory fibres coming from the $n$. perinealis, in a quantity somewhat larger than in the distal end of the vagina. The terminal formation of these fibres grows gradually more or less powerful as the distal end of the sinus is reached.

The nerve bundles coming from the $\mathrm{n}$. perinealis and reaching the ventrolateral side of the distal part of the vagina are made of many thick motor and sensory fibres and a small number of vegetative fibres, 
and while running through the surrounding circular striated muscle layer send off all their motor fibres into that layer. The attenuated bundles of sensory and vegetative fibres upon losing the motor fibres penetrate through the thick submucosa filled with venous plexus into the propria. Sensory fibres are seen also running into the poorly developed smooth muscle layer of circularly arranged muscle fibres during the course.

The sensory fibres in the propria are somewhat different in their development according the difference in the position of the mucous membrane. The positions where the sensory fibres are most adundant are the outer sides of the lateral edges of the semilunar lumen, next their inner sides, and the number diminishes widely in other positions. The terminations of the sensory fibres in the propria here comprise unbranched, branched and corpuscular types. Intraepithelial fibres are found in the epithelium and otherwise very peculiarly shaped branched terminations are found in the smooth muscle layer too.

The sensory fibres, upon reaching the propria, lose their myelin and pass over into their terminations. In the unbranched and the branched terminations, the fibres run winding courses peculiar to sensory fibres while undergoing changes in size and end in sharp- or bluntpointed terminal fibres just beneath the epithelium, but often enough, terminal fibres are found to run further up into the epithelium. For example, Fig. 7 shows a simple branched termination found in the

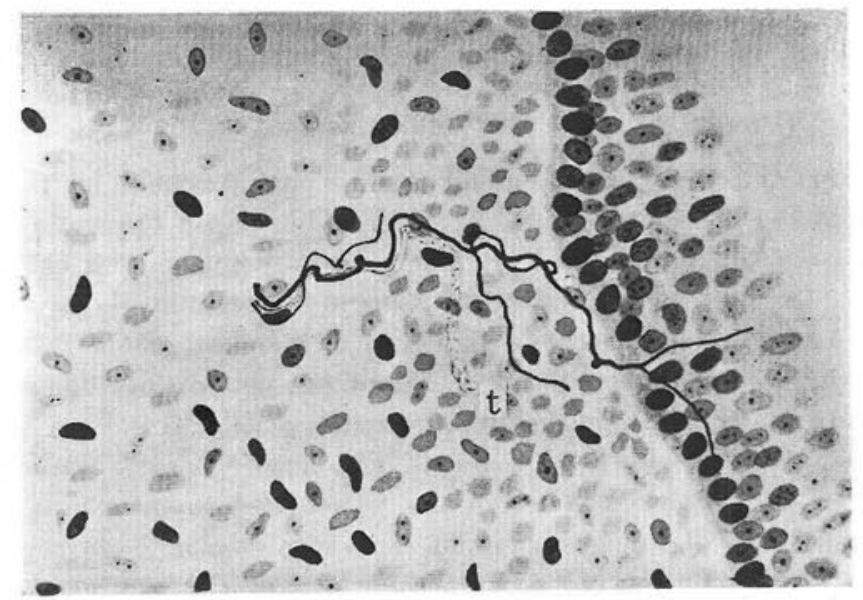

Fig. 7. A simple branched sensory termination found in the lateral wall of the distal part of the dog vagina. 2 terminal fibres run into the epithelium while the other one remains subepithelially. $t$ vegetative terminal reticulum. Same staining. $\times 400$. 
propria of the lateral wall of the vagina, and we find two of the terminal fibres runniug into the epithelium to end there. Frequently, such branched terminations are of rather complex formation. These are most often found at the lateral vertices of the semilunar lumen.

The intraepithelial fibres here may form unbranched as well as branched terminations. The nerve fibres composing such terminations too show changes in size during their courses and run not only between but also through the epithelial cells, to end sharply or bluntly or sometimes in small knobs. Not rarely too, the intraepithelial terminal fibres run up to the surface of the epithelium. Fig. 8 shows an unbranched

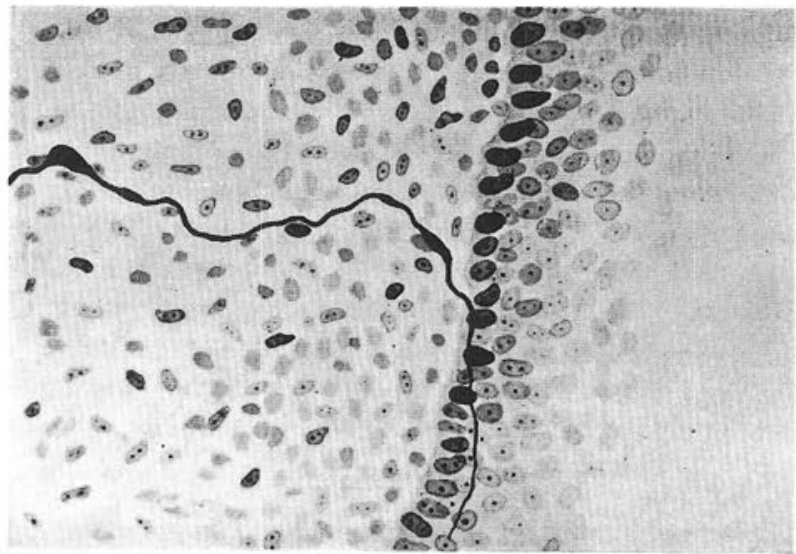

Fig. 8. An unbranched intraepithelial fibre found in the ventral wall of the distal part of the dog vagina. Details in the text. Same staining. $\times 400$, reduced to $2 / 3$.

intraepithelial termination found in the mucous membrane on the ventral side of the semilunar lumen. The stem fibre, upon losing its myelin, runs through the propria while undergoing very conspicuous changes in size into the epithelium and progressing circularly in its basal layer, ends in a sharp point therein. In Fig. 9 is shown a complex branched intraepithelial termination found at one of the vertices of the semilunar lumen. Here, the intraepithelial fibres are mostly fine ones showing little change in size in their courses, but one of them is rather stout and undergoes frequent change in size before it bifurcates into 3 short terminal fibres. The development of the above described intraepithelial fibres is also particularly good at the vertices of the semilunar lumen.

It is of deep interest that corpuscular terminations have been found in the propria mucosae of the distal part of the dog vagina, seeing that, according to the stndy by IKUI, such corpuscles have never been found in the distal part of the human vagina, a small number of simple 

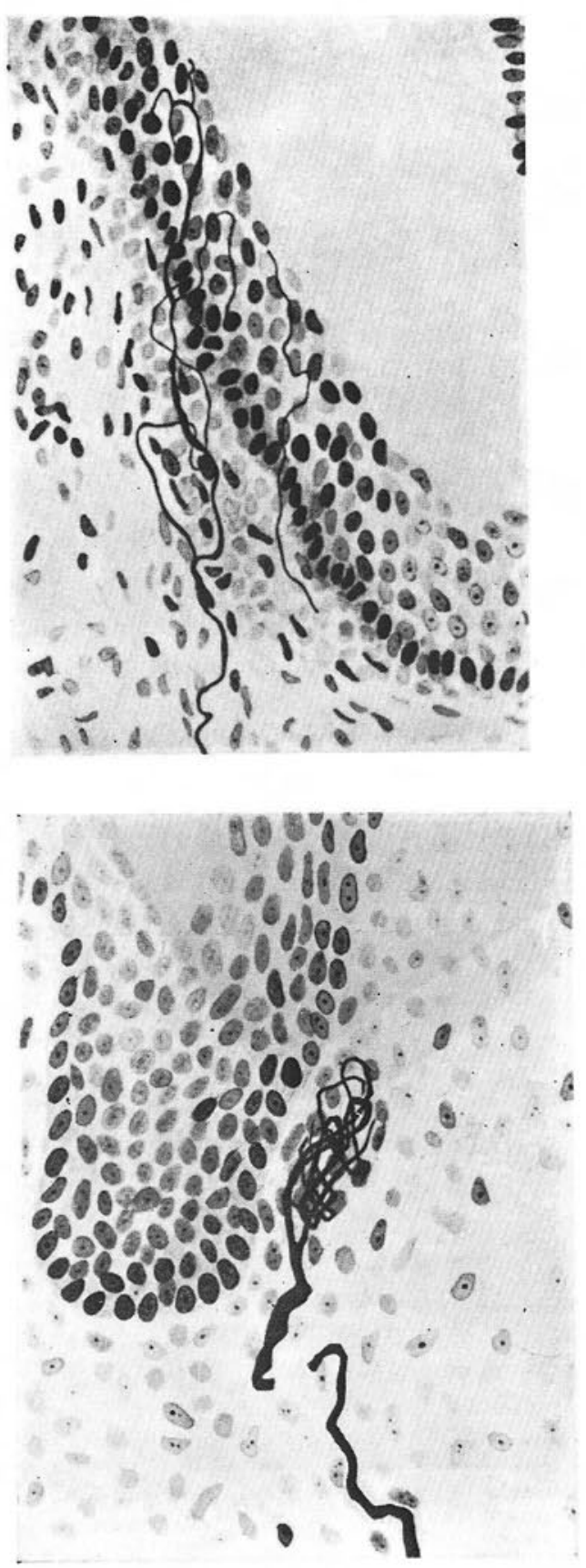

branched terminations only being found there. These corpuscular terminations in the dog vagina are of glomerular type and comprise both capsulated and uncapsulated ones, In Figs. 10 and 11 are illustrated uncapsulated glomerular terminations found in the propria at the vertices of the semilunar lumen. As particularly clearly illust.

Fig. 9. A complex branched intraepithelial sensory termination found in the lateral wall of the vertex of the semilunar lumen of the distal part of the dog vagina. Details in the text. Same staining. $\times 250$.

rated in Fig. 10, the stem fibre in this termination, after losing its myelin, shows conspicuous changes in size and soon divides into many branches which take ansiform courses and in some cases coming into mutual anastomosis, end in a glomerular arrange. ment as a whole. Many

Fig. 10. An uncapsulated glomerular termination originated in a very thick sensory fibre formed subepithelially in the lateral wall of the vertex of the semilunar lumen of the distal part of the dog vagina. Details in the text. Same staining. $\times 600$, reduced to $4 / 5$. 


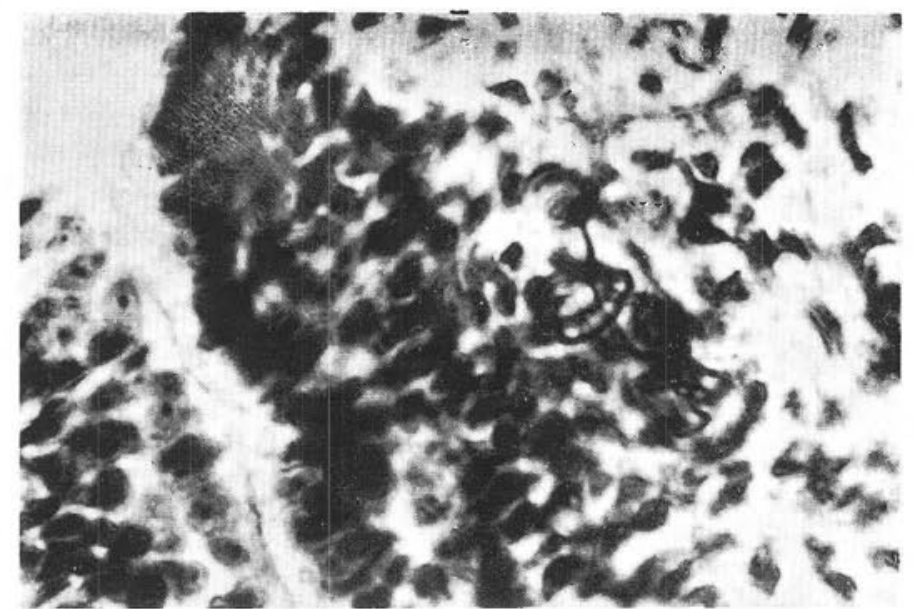

Fig. 11. An uncapsulated glomerular termination found in the inner wall of the same semilunar lumen. Photo $\times 600$.

specific nuclei seemingly derived from SCHWANN's nuclei are contained in these terminations.

Capsulated glomerular terminations, as shown in Figs. 12 and 13, are most freqnently formed in the small papillae protruding into the epithelium from the propria. This body is composed, as shown particularly clearly in Fig. 13, by $1-2$ thick fibres sending out several branches, which run peculiar winding courses arranged in a glomerulus

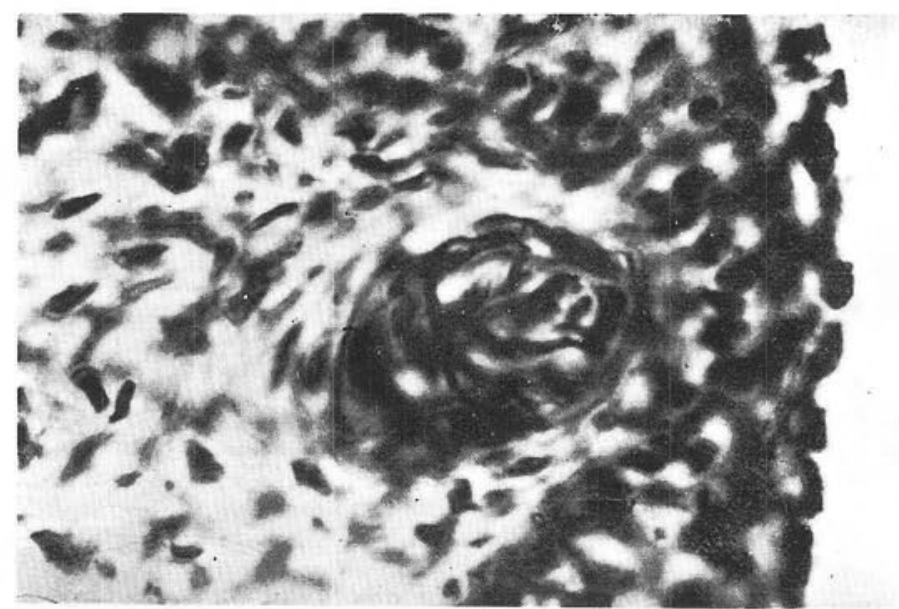

Fig. 12. A capsulated glomerular termination found in a small papilla of the lateral wall of the semilunar lumen of the distal part of the dog vagina. Same staining. Photo $\times 600$. 
in general form. Outwardly these bodies are covered by a typical connective tissue capsule and contain specific nuclei in them. The above corpuscular terminations are supposed to be related with the sexual feeling, as are the genital nerve bodies.

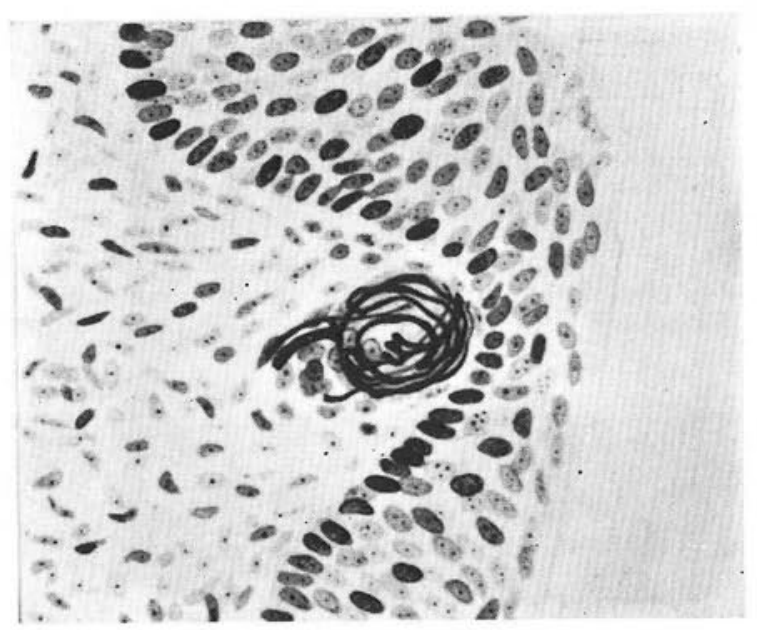

Fig. 13. A capsulated glomerular termination formed in a small papilla in the lateral wall of the semilunar lumen of the distal part of the dog vagina. Connective tissue capsule and specific nuclei are shown clearly. Same staining. $\times 300$.

In the ill-developed smooth muscle layer lining the inside of the striated muscle layer of the distal part of the dog vagina are frequently found some very peculiar branched sensory terminations. These terminations are found more frequently and more complexly formed in the urethra where this muscle layer is in better development. Fig. 14 shows a complex branched termiuation of such a peculiar type found in the well-developed smooth muscle layer of the urethra. The many branched fibres derived from the thick stem fibre run peculiar winding courses while undergoing frequent and peculiar change in size and end widely diffused in the muscle tissue. Most of the numerous terminal fibres are very thin and end in sharp points. Fig. 15 shows a simple branched termination found in a poorly developed circular smooth muscle bundle in the dorsal side of the distal part of the vagina. Here, a single stout stem fibre sends out several thin terminal branches, which run only short courses before ending in sharp points. This termination is of the same type as that in Fig. 14 but of a simpler formation.

Thus, branched sensory terminations are found in he smooth muscle layer of the distal parts of the urethra and the vagina of dog, and in 


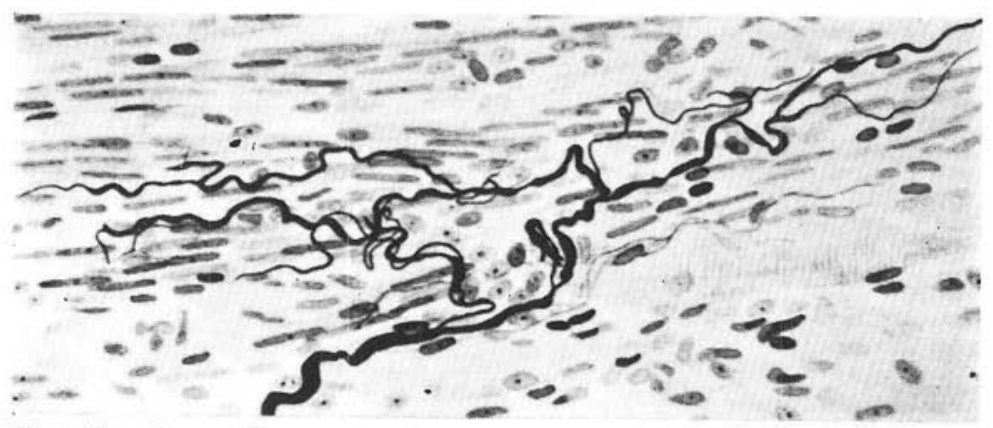

Fig. 14. A specific complex branched termination originated in a very thick sensory fibre found in the circular smooth muscle layer of the urethra of a femine dog. Details in the text. Same staining. $\times 250$, reduced to $4 / 5$.

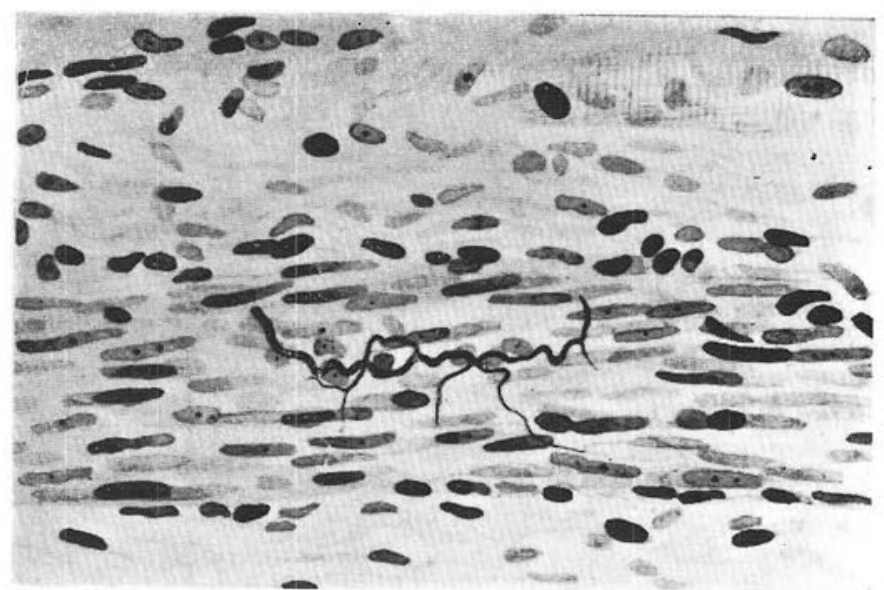

Fig. 15. A specific simple branched termination found in a circular smooth muscle bundle in the dorsal side of the distal part of the dog vagina. Details in the text. Same staining. $\times 300$.

the past there have been a few reports on similar findings in the muscle layers of the genitals. For example, NOBUTA has reported on the existence of branched terminations very similar to that shown in my Fig. 14 in the circular smooth muscle layer lining the inside of the striated muscle layer surrounding the ventral side of the bottom of the human prostata and more recently, MORI and OJIMA have described nearly identical branched terminations found in the innermost smooth muscle layer of the muscularis of the pars interglandularis and the pars membranacea of the urethra of male cats respectively. From the above findings, we may conclude that sensory terminations of peculiar shape 
are found formed in the muscularis, especially the smooth muscle layer of the outer genital canals. The function of these terminations is not too clear either, but it may be that these are also related with the sexual feeling, as the corpuscular terminations above.

In the initial part of the sinus urogenitalis, the lumen is semilunar in its cross-section resembling that of the distal part of the vagina, and the histological picture of this part is also similar to that of the latter. Consequently, the distribution of the sensory fibres and their terminations here is not much different from that of the latter either, except that the number and the development of these elements become seemingly somewhat superior upon coming into the sinus. But going more caudal and the middle one-third of the sinus being reached, the sensory fibres become considerably numerous and their terminations increase in complexity, corpuscular ones resembling the so-called RUFFINI's bodies being frequently found, besied the above glomerular terminations.

In Figs. 16 and 17 are shown unbranched and simple branched termi-

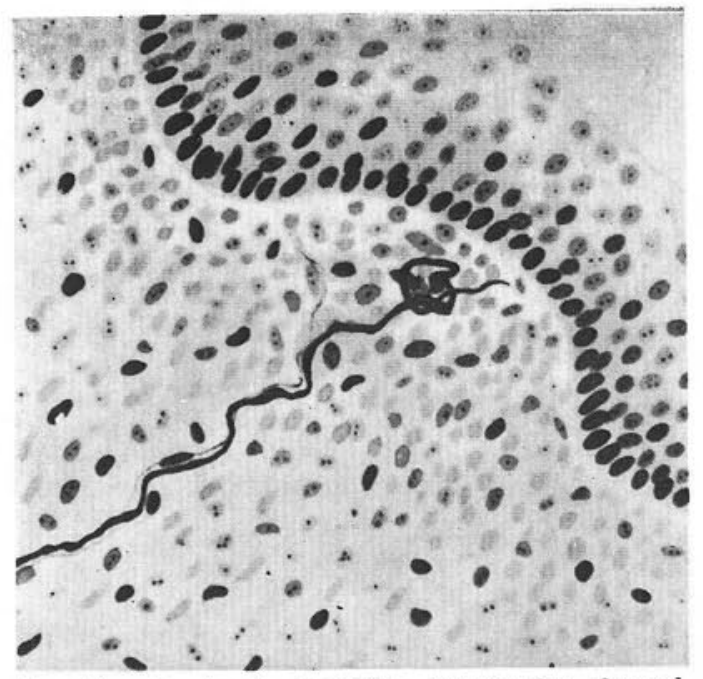

Fig. 16. A simple loop-like termination formed subepithelially in the lateral wall of the mid-part of the sinus urogenitalis of a femine dog. Same staining. $\times 300$, reduced to $4 / 5$.

nations found in the propria of the lateral wall in the midpart of the sinus. As is made clear in the figures, in these terminations, very many of the terminal fibres end in deseribing various forms of loops with their extremities. Such terminations were not frequent in the proximal part of the sinus or the distal part of the vagina and show a 


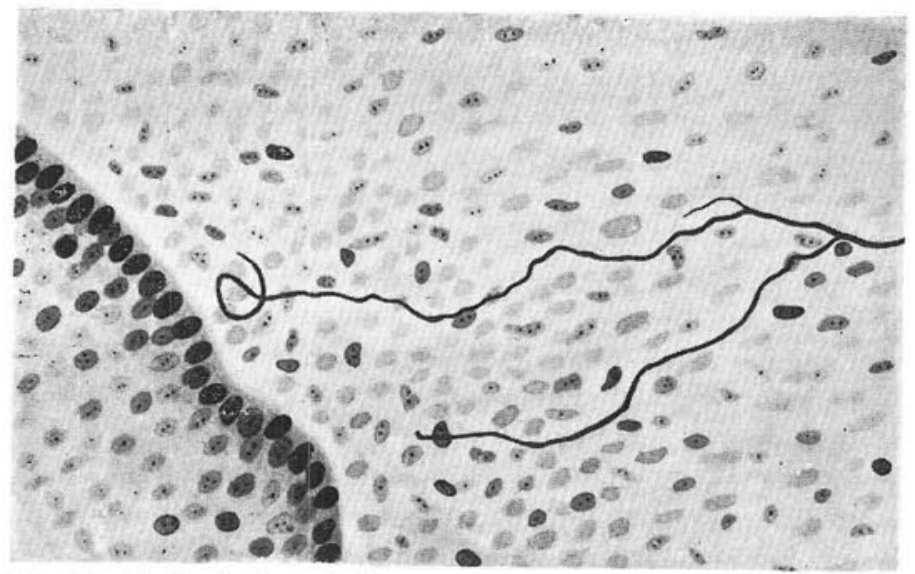

Fig. 17. A simple branched termination found in the propria of the lateral wall of the mid-part of the sinus urogenitalis of a femine dog. One of the terminal fibres ends in a simple loop. Same staining. $\times 400$, reduced to $4 / 5$.

more complex course their terminal fibres can take. Branched terminations of complex type are indeed found here, but not in quantity.

Intraepithelial sensory terminations are rather abundant also in the mid-portion of the sinus, but complex typed ones are comparatively rare

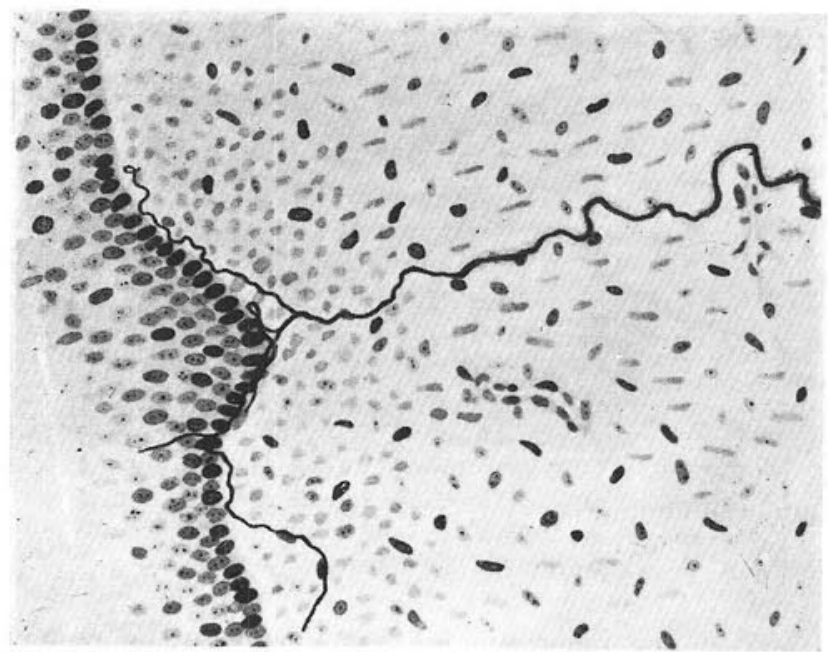

Fig. 18. A simple branched termination found in the lateral wall of the mid-part of the sinus urogenitalis of a femine dog. 2 of the terminal fibres pass over into intraepithelial fibres. Details in the text. Same staining. $\times 300$, reduced to $4 / 5$. 
here. A simple branched termination found in the lateral wall is illustrated in Fig. 18. A rather thick fibre, upon losing its myelin, runs up toward the epithelium to branch out into 4 rami, of which 2 run up further into the epithelium to end as intraepithelial fibres, while the other 2 end bluntly just beneath the epithelium.

The glomerular terminations, one of the classes of corpuscular terminations found in the mid-part of the sinus, comprise both the

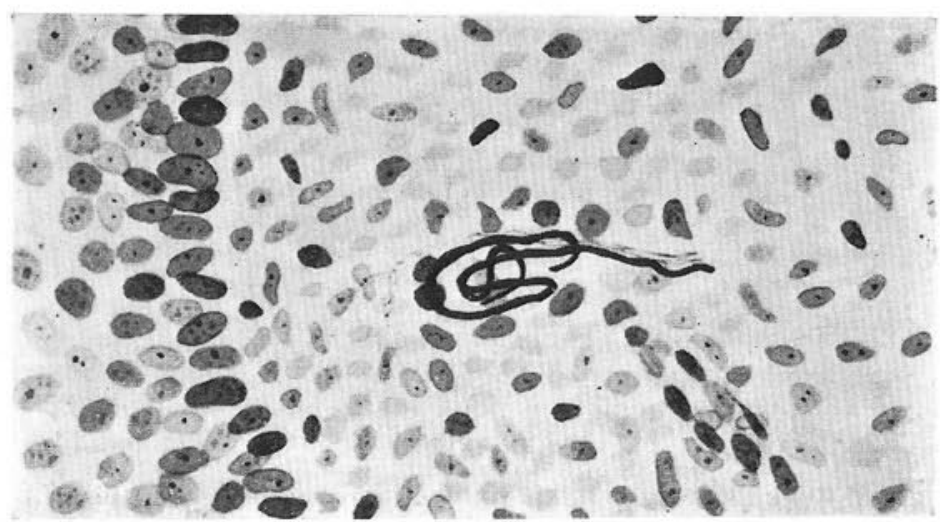

Fig. 19. A small-sized uncapsulated glomerular termination formed subepithelially in the ventral wall of the mid-part of the sinus urogenitalis of a femine dog. Same staining. $\times 400$.

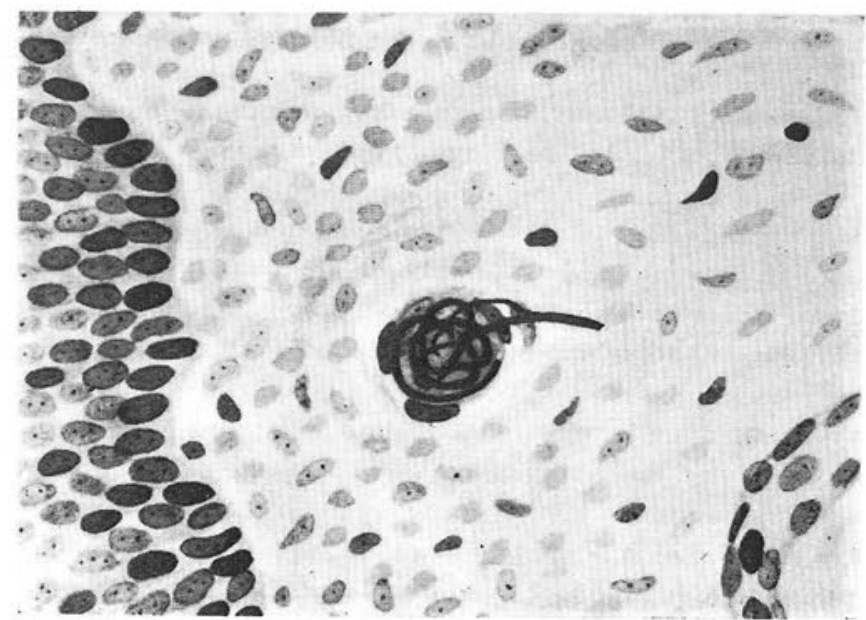

Fig. 20. A small-sized capsulated glomerular termination formed subepithelially in the dorsal side of the mid-part of the sinus urogenitalis of a femine dog. Connective tissue capsule and speci. fic nuclei are shown clearly. Same staining. $\times 300$. 
capsulated and the uncapsulated types. In size and number, however, these are rather small. Figs. 19 and 20 show subepithelially formed uncapsulated simple and capsulated glomerular terminations respectively.

The other type of corpuscular terminations comes forth rather frequently. As shown in Figs. 21 and 22 , these consist of cylindrical or oval capsulated terminations found in the propria, with an inner bulb

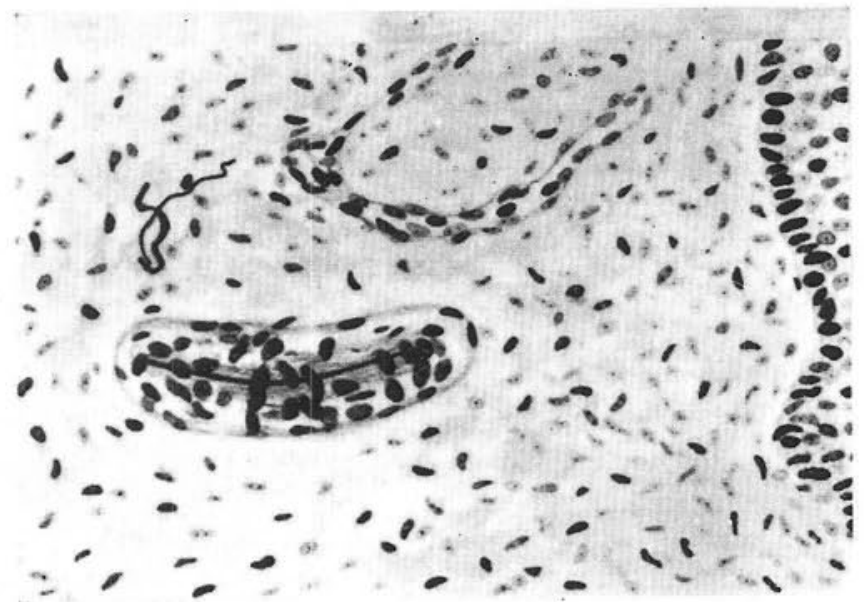

Fig. 21. A cylindrical capsulated specific end body in which the inner bulb is filled with fine parallel fibres at the center found in the propria of the lateral wall of the mid-part of the sinus urogenitalis of a femine dog. Details in the text. Same staining. $\times 300$, reduced to $4 / 5$.

of very interesting structure. In such a termination, many very fine fibres running parallel to the longer axis are seen at the center, surrounded by oval or round specific nuclei arranged rather sporadically and a connective tissue capsule containing narrow nuclei covers up this formation. In general, a single thick sensory fibre runs into the bulb through one of its poles after losing its myelin and ends bluntly or sometimes sharply near the opposite pole (Fig. 21). What is of the highest interest about such terminations is the existence of many parallel fibres in the inner bulb. Since these fibres often reach into the periphery of the inner bulb (Fig. 22), such a termination may give an impression similar to a PACINIan body, but a nearer examination reveals the absence of the club-like inner bulb rich in nuclei usually seen in a PACINIan body and it is clear that it is distinct from such a body. Terminations resembling such corpuscles have been found by KATO not rarely in the dog glans clitoridis. He has opined that these might be RUFFINIan bodies, to which opinion I heartily comply. I 


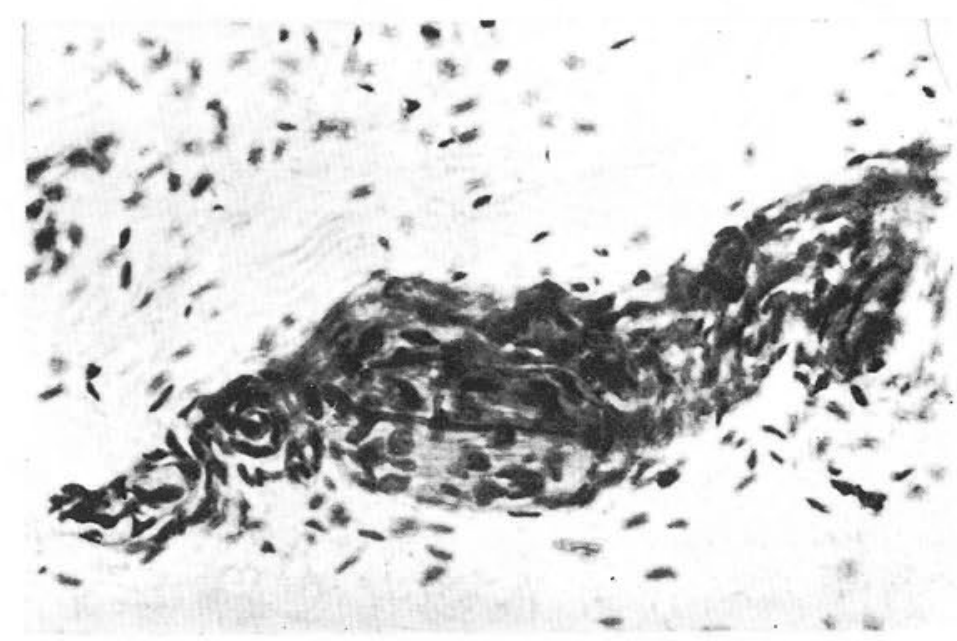

Fig. 22. An oval capsulated specific end body in which the inner bulb is all over filled with fine parallel fibres found in the dorsal wall of the mid-part of the sinus urogenitalis of a femine dog. Details in the text. Same staining. $\times 200$.

believe that these bodies are related with the sexual feeling, too.

The sensory innervation of the distal part of the sinus urogenitalis is scarcely different from that of the middle one-third of the sinus, perhaps except only in the slightly large number of the sensory fibres in the former.

\section{Summary.}

The distal portion of the sinus urogenitalis of dog has a split-formed lumen elongated dorsoventrally, but toward the mid-part, it loses in height and the lumen becomes rhomboid in cross-section, and in the more cranial part of the sinus, the lumen becomes semilunar in cross-section, with its concave side facing ventralwards. In the distal part of the vagina, the curvature of the crescent-shape becomes more and more heightened and the lumen becomes more and more narrow. More cranialwards the vagina attains its proper structure, the curvature of the semilunar cross-section becoming gentler and many folds being formed in the mucous membrane by furrows dug into it from the side of the lumen. In the more cranial part, the lumen becomes quite straight and horizontal in cross-section and the formation of the folds and the furrows becomes more accentuated. Viewed histologically, the distal end of the vagina forms the beginning of the sinus urogenitalis.

The lumina of the vagina and the sinus urogenitalis show a great morphological change by positions but the superficial area of the mucous 
membrane remains nearly unchanged between the distal end of the vagina and the same of the sinus, while that of the mucous membrane of the vagina proper is about $3-4$ times as broad as that in the parts above.

The morphological changes in the lumina of the vagina and the sinus urogenitalis are found to accompany corresponding histological difference, which has a close bearing on their sensory innervation.

From the vagina proper down to the distal end of the sinus urogenitalis, the epithelium and the propria show little change, but the submucosa is found to contain no venous plexus in the vagina proper, but a large quantity of $j t$ in the distal part of the vagina. This plexus, however, diminishes again slowly as the distal end of the sinus is approached. The muscularis is very well developed in the vagina proper, having a strong circular striated muscle layer lining its outside and bornded on the ventral side by an adventitia from the urethra. In the distal part of the vagina, the muscularis becomes abruptly poorer, remaining only as small circular bundlets lining the Inside of the equally altenuated striated muscle layer. It loses further in development as the sinus is approached and soon vanishes altogether. When the urethra becomes absorbed in the tissue of the distal part of the vaginal wall, its muscularis once so strongly developed loses suddenly in strength and its submucosa fuses with that of the vagina.

If the sinus urogenitalis in the outer genitals of dog described above, where the vagina and the urethra run together, can be identified with the human vestibulum vaginae in nature, the sensory innervation of these parts of dog is not much different from that in man. As in man, the vagina proper in $\operatorname{dog}$ is not sensorily innervated, but in its distal part, we see some sensory fibres from the $\mathbf{n}$. perinealis running in and the sinus urogenitalis is somewhat richer in sensory innervation.

The sensory terminations in the distal part of the vagina are larger in number and generally more complex in structure in tog than in man. In the propria there, beside unbranched and branched terminations, a small number of glomerular terminations, either capsulated or not, are found in existence, and unbranched and branched terminations are formed also in the epithelium. Further, a comparatively large number of peculiar branched terminations are found in the smooth muscle layer lining the inside of the striated muscle layer. Complex ones of these peculiar terminations are observed in a rather large quantity also in the smooth muscle laver of the urethra adjacent to the vagina proper.

In the proximal portion of the sinus urogenitalis we find sensory terminations approximately identical with those in the distal part of the vagina in existence, but in the middle one-third of the sinus, beside the various types of terminations above, I have found also corpuseular ter- 
minations resembling RUFFINIan bodies here and there. This state of sensory innervation continues unchanged down to the distal end of the sinus urogenitalis.

\section{內 容自 抄.}

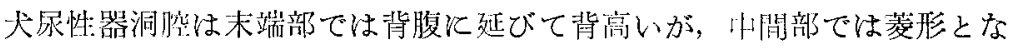

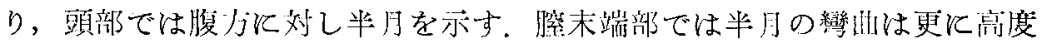

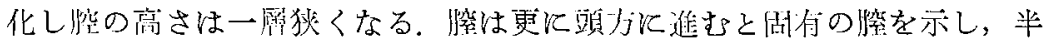

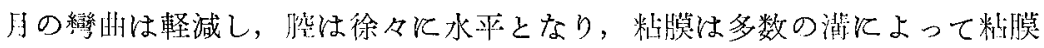
版壁の形成を示す。

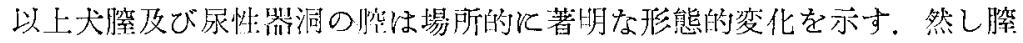

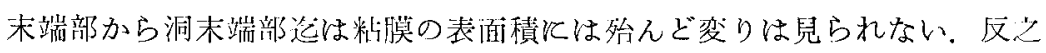

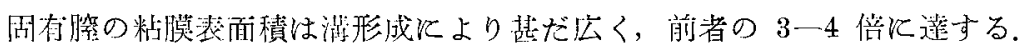

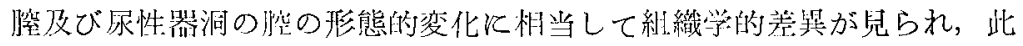
事は其知敩神経分布に車大閣係を示す。

湖有腔から尿性器洞の末端部に至る迄上皮と固有脱には大した变化は見

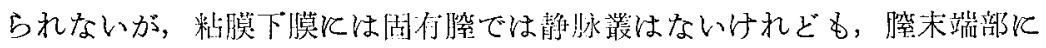

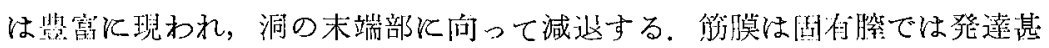

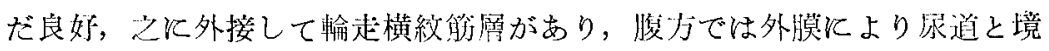

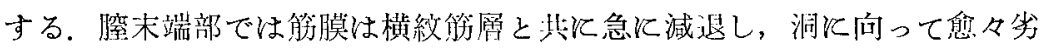

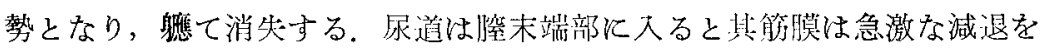
示し，粘膜下膜は幥の夫炕融合する。

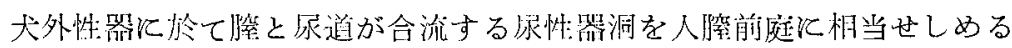

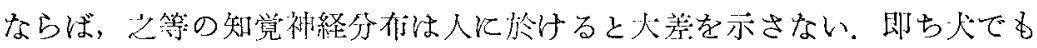

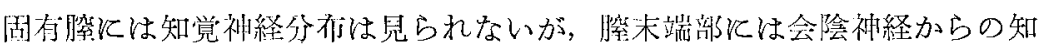

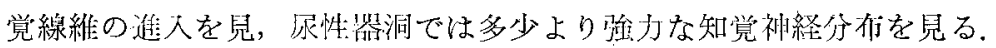

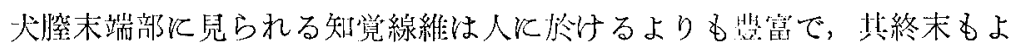

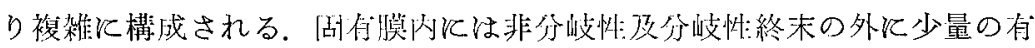

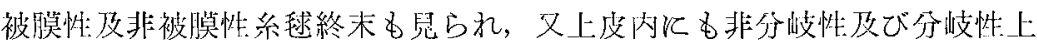

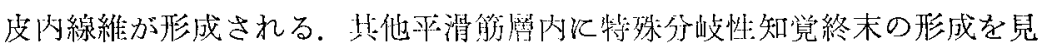

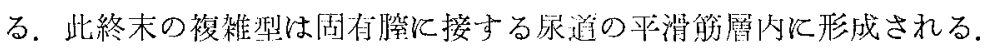

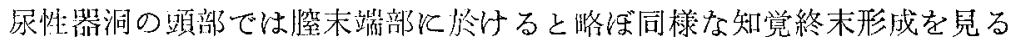
が，洞山部では其他 Ruffini 氏小俭江類似の終末が所々に発兒される。然

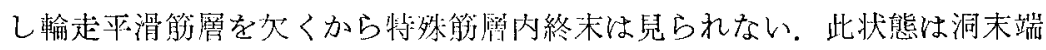
部まで続く。 


\section{References.}

Ikui : Tohoku Igaku Zassi. (Jap.) 39 (1949). - Kato : Arch. hist. jap. 9 (1955). - Mori : Arch. hist. jap. 8 (1955). - Nobuta : Tohoku Igaku Zassi. (Jap.) 39 (1949). 一 Ojima: Arch. hist. jap. 10 (1955). 